\title{
BMJ Open Perspectives of health professionals towards deprescribing practice in Asian nursing homes: a qualitative interview study
}

Chong-Han Kua, ${ }^{1,2}$ Vivienne SL Mak, ${ }^{3}$ Shaun Wen Huey Lee (iD) ${ }^{1,4}$

To cite: Kua C-H, Mak VSL, Lee SWH. Perspectives of health professionals towards deprescribing practice in Asian nursing homes: a qualitative interview study. BMJ Open 2019:9:e030106. doi:10.1136/ bmjopen-2019-030106

- Prepublication history for this paper is available online. To view these files, please visit the journal online (http://dx.doi org/10.1136/bmjopen-2019030106).

Received 27 February 2019 Revised 26 August 2019 Accepted 25 September 2019

Check for updates

(c) Author(s) (or their employer(s)) 2019. Re-use permitted under CC BY-NC. No commercial re-use. See rights and permissions. Published by BMJ.

${ }^{1}$ School of Pharmacy, Monash University—Malaysia Campus, Bandar Sunway, Malaysia ${ }^{2}$ Continuing and Community Care Department, Tan Tock Seng Hospital, Singapore, Singapore ${ }^{3}$ Centre for Medicine Use and Safety, Faculty of Pharmacy and Pharmaceutical Sciences, Monash University_Parkville Campus, Parkville, Victoria, Australia

${ }^{4}$ School of Pharmacy, Taylor's University, Subang Jaya, Malaysia

Correspondence to Chong-Han Kua; chong.kua@monash.edu

\section{ABSTRACT}

Objective To examine the determinants of deprescribing among health professionals in nursing homes, focusing on knowledge, practice and attitude.

Design This was a qualitative study comprising semistructured face-to-face interviews guided by 10 open-ended questions. Interviews were conducted until data saturation was achieved and no new ideas were formed. The interviews were audio-recorded, transcribed verbatim and analysed for themes. To derive themes, we employed directed content analysis of transcript data. Coding was completed using a combination of open, axial and selective coding.

Setting Four nursing homes in Singapore.

Participants The study involved 17 participants (comprising 4 doctors, 4 pharmacists and 9 nurses). Results Two key themes were identified, enablers and challenges. These were enablers and challenges faced by doctors, pharmacists and nurses towards deprescribing. The identified subthemes for enablers of deprescribing were: (1) awareness of medications that are unnecessary or could be targeted for deprescribing; (2) improving quality of life for patients with limited life expectancy; (3) improving communication between doctors, pharmacists and nurses; (4) systematic deprescribing practice and educational tools and (5) acknowledgement of possible benefits of deprescribing. The identified subthemes for challenges of deprescribing were: (1) symptoms not acknowledged as possibly drug-related; (2) lack of knowledge in patient's and family members' preferences; (3) lack of coordination between health professionals in hospitals and nursing homes and (4) limited tools for deprescribing. The development of a local guideline, mentoring nurses, case discussions, better shared decision-making and improving multidisciplinary communication, may help to support the process of deprescribing.

Conclusion In conclusion, this study highlighted that deprescribing in the nursing homes is perceived by health professionals to be challenging and future research could assess how routine case studies, mentoring and better multidisciplinary communication could improve deprescribing knowledge and process in the nursing homes.

\section{INTRODUCTION}

Many nursing home residents have advanced frailty and confusion. ${ }^{1}$ Older adults residing in nursing homes often have multiple

\section{Strengths and limitations of this study}

- We studied deprescribing practices by conducting interviews in an under-represented setting; Asian nursing homes.

- This study provided important insights and areas for improvement in the process of deprescribing in nursing homes.

- As the study was only conducted in Singapore, findings may not be representative of other Asian countries and settings worldwide.

comorbidities requiring nursing care. ${ }^{2}$ As a result, they are often prescribed multiple medications, leading to a high prevalence of polypharmacy (commonly defined as five or more medications). ${ }^{3}$ Polypharmacy is associated with an increased risk of negative health outcomes including adverse drug events, drug interactions, decreased functional status, geriatric syndromes, higher healthcare costs and non-adherence. ${ }^{4}$ Medication management for these residents is further challenged by multiple healthcare providers, hospital admissions, rigid organisational structures, resource limitations, medical hierarchies, contrasting care expectations of family and doctors and the variable life priorities of each individual resident. $^{6}$

There is evidence that deprescribing, or the process of reducing, tapering and discontinuing inappropriate or unnecessary medications among older adults can potentially improve patient outcomes. ${ }^{78}$ Deprescribing in nursing homes can reduce the number of residents with potentially inappropriate medication by $59 \%$, the number of residents who have experienced a fall by $24 \%$ and mortality by $26 \% .^{9}$ As such, an understanding of the enablers and challenges to deprescribing among health professionals is essential to facilitate successful deprescribing interventions. 
Several studies have explored the perceptions, enablers and challenges of general practitioners (GPs) and other health professionals towards deprescribing. ${ }^{6}{ }^{10-12}$ These studies found that challenges to deprescribing included existing organisation systems and policies, self-perceived restriction in the ability to be involved in medication-related issues, lack of knowledgeable and skilled personnel as well as attitudes (including devolving of responsibility between GPs and specialist physicians). ${ }^{6} 11$

There are varying priorities between the professions on factors that are important for deprescribing in long-term care facilities. Some of the key considerations include: 'evidence for deprescribing', 'clinical appropriateness of therapy' as well as 'clinician receptivity', with different behaviours and attitudes reported between countries. ${ }^{12}{ }^{13}$ For example, Swedish GPs expressed that their main concern in medication management was to achieve a good quality of life, while among Australian GPs, they were more concerned with the low financial reimbursement associated with providing care to these residents. ${ }^{13}$

Although there was numerous literature that explored the perceptions, enablers and challenges of health professionals towards deprescribing, there is a limited understanding of the perspectives of health professionals towards deprescribing in nursing homes, particularly in Asia where the concept of deprescribing is still relatively new and the populations are rapidly ageing. Previously, a qualitative meta-synthesis of enablers and challenges of doctors towards minimising potentially inappropriate medications (PIMs) in community older adults identified analytical themes intrinsic to the prescriber (beliefs, attitudes, knowledge, skills, behaviour). ${ }^{14}$ These include problem awareness, inertia secondary to lower perceived value proposition for ceasing versus continuing potentially inappropriate medications and self-efficacy in regard to personal ability to alter prescribing, from which enablers and challenges to minimising PIMs emerged. Therefore, in order to develop processes of deprescribing within a particular healthcare system, it is vital to gain an understanding of the enablers and challenges pertinent in developing the right processes that lead to successful uptake of deprescribing.

\section{Aims}

The aims of this study are to examine the factors that affect the views and acceptance of deprescribing among health professionals in nursing homes, focusing on knowledge, practice and attitude.

\section{METHODS AND ANALYSIS}

This was a qualitative study with doctors, pharmacists and nurses to determine the factors that affect their views and acceptance of deprescribing in Singapore nursing homes. Semi-structured interviews were conducted prior to the implementation of a deprescribing stepped-wedge randomised controlled study. ${ }^{15}$
Participants had to satisfy the following inclusion criteria: (1) provide informed consent and (2) be involved in the care of nursing home residents. Participants could opt out at any time during the study. We did not apply any other inclusion criteria due to the limited numbers of doctors and pharmacists across the four participating nursing homes. Doctors and pharmacists were approached by the principal investigator (C-HK) at the study sites during their routine visits. Convenience sampling of nurses across the four homes was employed until data saturation was achieved.

\section{Participants and settings}

The interviews were conducted in four nursing homes (one with approximately 400 beds, two with 200 beds and one with 150 beds) across Singapore. The pharmacists were community-based pharmacists who provide weekly or fortnightly medication review services to the residents for at least the past year. These pharmacists were either in progress or have completed a postgraduate study (ie, Master of Clinical Pharmacy) or board certification in geriatric training. Nurses were full-time employees (staff nurses or enrolled nurses) of the nursing homes. Doctors were GPs who provided clinical services at the nursing homes at the time of the interview. Most doctors visited the homes at least once weekly or fortnightly.

\section{Semi-structured interviews}

All interviews were conducted in a private room (nurse's office or doctor's consultation room) within the nursing homes at a time convenient for each participant. The principal investigator, C-HK, conducted all interviews. The interview was guided by 10 open-ended questions on knowledge, practice and attitude (KAP) towards deprescribing (box 1) and was qualitatively analysed using thematic analysis. The KAP conceptual framework was employed in this study. The questions were developed by expert opinions between the researchers (C-HK, SWHL, $\mathrm{VM}$ ) and a senior consultant geriatrician working in the settings. The interview was piloted on a doctor, a pharmacist and a nurse to determine the clarity and comprehensibility of the questions, as well as the time taken to complete the interview. No changes were required to the original interview questions.

\section{Data analysis}

Each interview was audio-recorded and transcribed verbatim with the participant's consent. We used QSR NVivo 11 to assist in analysis of the data. Both an inductive and deductive approach were used to explore both intended issues and other unexpected aspects of participants' experience. ${ }^{16}$ In conventional content analysis (inductive approach), we assessed the various clinical characteristics of the doctors, pharmacists and nurses across the four nursing homes in general (such as primary place of practice, any specialisation, length of practice in nursing homes, any access to education infrastructure). These were used to develop themes and a coding scheme. 


\section{Box 1 Interview questions}

\section{Knowledge}

1. Which type of medications do you think should be deprescribed in elderly?

2. Do you think under-reporting of possible adverse drug events by attributing to old age is common and why?

3. Do you use or feel a need for guidelines for deprescribing and why? If you are using guidelines, which are you aware of and which edition?

\section{Practice}

1. Do you think taking medications to prevent diseases are necessary and why?

2. Do you think nurses, doctors and pharmacists have to work together in deprescribing practice and why?

3. Do you consciously practice deprescribing?

4. Do you take into account of your patients' preference in treatment selection?

\section{Attitude}

1. Do you think deprescribing is important and in which aspect/s you can think of?

2. If you are already practising deprescribing, how do you think you can do it better?

3. If you are not practising deprescribing, what will increase your confidence in doing it?

Following which, we employed directed content analysis (deductive approach) to collate qualitative data and the transcript data placed into themes. Coding was done using a combination of open, axial and selective coding. Reporting of this manuscript followed the Standards for Reporting Qualitative Research reporting guidelines. ${ }^{17}$

\section{Patient involvement}

Patients and the public were not involved in the design or planning of the study.

\section{RESULTS}

\section{Study participants}

Nineteen participants were approached for the interview and all agreed to participate. However, two (a pharmacist and a doctor) declined consent for recording and their data were not included in the study. The interviews lasted 14 min on average. Participants comprised four doctors, four pharmacists and nine nurses. Eleven $(64.7 \%)$ of the participants were female. No other demographic profile was collected due to confidentiality concerns of the nursing homes.

Two key themes (enablers and challenges) were identified in the interviews (table 1).

\section{Theme: enablers to deprescribing ( $D=$ doctor, $N=$ nurse, $\mathrm{P}=$ pharmacist)}

\section{Subtheme: awareness of medications that are unnecessary or could be targeted for deprescribing}

Acceptance of participants towards deprescribing is facilitated by an increased awareness of the medications that are unnecessary or inappropriate (poor risk-benefit profile) for older patients. Pharmacists and doctors primarily viewed gastroprotective agents (proton pump inhibitors, histamine-2 receptor antagonists) as unnecessary medications. This may be due to a previous local awareness campaign to deprescribe proton pump inhibitors in Singapore. ${ }^{18}$ Other types of medication viewed as potential targets for deprescribing include medications with high-risk profiles, such as sedative first-generation antihistamines and benzodiazepines. There was an emphasis from doctors on the risk-benefit ratio of the medication to be considered for taking off.

...medicine that does not benefit the patient or there is the poor risk-benefit profile. These are the medicine that I think should be deprescribed. (D10, male)

In contrast, nurses perceived supplements such as multivitamins, iron, calcium and glucosamine as targets for deprescribing.

Oral hypoglycaemic agents and antihypertensives were also viewed by some doctors and nurses as the focus for deprescribing. For some patients, dietary plans provided within nursing homes (moderate salts and sugar) were sufficient to control the patients' medical conditions.

In addition, one participant identified medications such as statins and bisphosphonates that require a longer time to achieve its outcomes as unnecessary.

If the medication takes a longer (time to see clinical benefits)...you see the effect only after years, I think

Table 1 Themes and subthemes

\begin{tabular}{ll}
\hline Themes & Subthemes \\
\hline Enablers of deprescribing & 1. Awareness of medications that are unnecessary or could be targeted for deprescribing. \\
& 2. Improving quality of life for patients with limited life expectancy. \\
& 3. Improving communication between doctors, pharmacists and nurses. \\
4. Systematic deprescribing practice and educational tools. \\
5. Acknowledgement of possible benefits of deprescribing.
\end{tabular}


there is no point to have them on...those osteoporosis medications, bisphosphonates, etc. (P19, female)

\section{Subtheme: improving quality of life for patients with limited life expectancy}

The life expectancy of older patients was a consideration by all groups to deprescribe. Most participants felt that deprescribing is important in an older patient with limited life expectancy, as there is a lack of evidence of clinical benefits from certain classes of medications.

If the patient's life expectancy is not too great and most of them are already on the advanced care plan. Then of course, all of these preventive medicines, we do not really need them. Whether I actively remove the one, it depends case by case. A patient has a lot of pill burden...then, yes, I would actively try to deprescribe. But I think that sometimes, the patient does not have a lot of medicine. They might be on some preventive ones...(so) leave it. (P12, female)

A pharmacist explained that she would not actively recommend the addition of medications, as quality of life was also an important consideration for older patients.

But if he is taking 10 to 20 years, I think it (deprescribing) is...giving quality of life to the patient. They are eating a lot of medication. (N8, female)

Lifetime cost and functional status were important factors for doctors in deciding whether to commence or stop a medication.

\section{Subtheme: improving communication between doctors, pharmacists and nurses}

And also the doctor as... a team to practice it (deprescribing). But currently, I just... review the patient individually. (P15, female)

... is good if they can work as a team...basically if they have a common understanding. (D5, male)

Most participants agreed that team communication was important in deprescribing, as doctors manage patient's overall condition, while pharmacists are medication experts and nurses are able to monitor its side effects and efficacy. One doctor felt team communication was not needed as those medications being deprescribed were considered non-essential medications. On the other hand, nurses felt that pharmacists were important to help re-evaluate what doctors and nurses missed out. Teamwork between doctors, pharmacists and nurses can be strengthened by improving communication, working towards an aligned care management care plan for older adults and ensuring its continuity.

Yes, because the nurses are the closest ones to the patients, so they can actually tell you if the medications are working or not and if there is any side effects... better than anyone else. Pharmacists obviously being the drug expert, have an obvious role to play in suggesting which medications can be deprescribed. And you need the doctors' help to deprescribe them because we do not have the power to stop them. (P19, female)

...because in this medical field, we really need collaboration. Teamwork...because the doctors are not here always. (N13, male)

\section{Subtheme: systematic deprescribing practice and educational tools}

Participants suggested that a more systematic guideline, clear-cut algorithm and multidisciplinary efforts were needed to ensure understanding and smoothen the process.

A standard guideline that would help, because we have so many pharmacists with different ways of practicing and different habits. So it would be better if we have something standardized to follow. So that all (nursing) homes can have the same, sort of, deprescribing procedures. (P12, female)

And where is the guide...you see, there is actually no clear guideline...local guidelines. The expert opinion...more specific guidelines with regard to certain medication, common medication that would be useful. (D5, male)

I think guideline...If there is a clear-cut algorithm... pharmacists are (taught to follow) algorithm... So we love algorithm. (P1, female)

Also, participants suggested other areas of improvement including face-to-face doctor-pharmacist discussions, as well as a deprescribing quick reminder guide.

I think...discussion...sometimes...where we intervene...the deprescribing, maybe we missed out some of the important information. For example, we are not aware of the latest condition but doctor (does, he is) the one who also work closer with the nurse and... the family. Doctor also examine the patient regularly that is why doctor will know more about the patient. (P15, female)

...like small cards, a reminder to try to cut-off PPIs (proton pump inhibitors), if there is no clear indication. Because a lot of current usage has...unclear indication. If now they have this very thick standard, black and white thing (guidelines)...pharmacists are more confident in cutting down medications. (P12, female)

Additionally, nurses noted that mentoring, case studies, lectures and guidebooks would be useful to get more nurses to participate in deprescribing.

I think those senior ones will not have much of a problem; they know their medication...these are for the junior (nurses)...Mostly they just follow the orders, until they get to the stage where they can mostly be on their own. (N8, female) 
So...we will talk about the resident's condition and if he benefits (from) the medicine, or if he does not benefit (from) the medicine so we can off it...(for example) the case study. (N6, male)

lecture plus...booklet so that...easy to pick up. (N4, male)

\section{Subtheme: acknowledgement of possible benefits of deprescribing}

Most felt deprescribing was important to reduce pill burden, adverse drug reactions, drug interactions, medication cost, medication errors and improve quality of life. In addition, burden to the healthcare system was also frequently brought up.

One, it (deprescribing) reduces and side effects... Two, it reduces pill burdens...the cost...It also reduces manpower...And with less...medication error. (D11, female)

... reduces the cost...maybe side effect. (N7, female)

Sometimes...They have been spending for years (on) medication but, it is not useful to the health condition, right. (N14, male)

$\ldots$ if there are a lot of drugs, and certain drugs that they decided to reduce or increase...(when) it come in blister packets...it is really tedious to actually open and then change the drugs. Yes, it is very time-consuming. (N17, female)

\section{Theme: challenges to deprescribing}

\section{Subtheme: symptoms not acknowledged as possibly drug- related}

Generally, pharmacists and doctors felt that adverse drug events often went undetected as symptoms were not acknowledged as possibly drug-related and therefore lacked acknowledgement that deprescribing was possible. Many patients have poor cognitive status (eg, dementia), physical status (eg, immobile or bedridden) or difficulty in communication, rendering them unable to inform and report any adverse events.

Those patients are...unaware that these are side effects of the medication. They think that...these are just part of aging... they do not think that there was have any alternative...And probably...family also have some of these perspectives. So sometimes even if they complain, (the) family will...simply brush off (as) just part of aging. (D11, female)

Nurses, on the other hand, felt that under-reporting was uncommon as they are around the patients most of the time but do agree that symptoms like dizziness may be hard to detect as these were multifactorial and can be precipitated by poor diet. One doctor also thought that under-reporting could be due to the nurses' lack of knowledge on the side effects of medications.
Subtheme: lack of knowledge of patient's and family members' preferences

Most health professionals would take into account the patient's condition (such as the ability to swallow) and cost, more than the patient's personal preference in deciding medication choice. Whether the patient can communicate to the doctors and nurses also played a big role in letting the patient decides. However, health professionals were often unable to assess the patient's preference due to their speech or cognitive disabilities and difficulties in contacting their family members. Pharmacists tend to go with the nurses' feedback rather than the patient's preference as mentioned in the interviews.

Yes, but I think that in this nursing home setting, a lot of the patients are not able to give preference, or it could be the family's preference...I guess, it is more like, if (the) patient is tube feeding, then I will take into account what dosage forms are more suitable for that route of the administration. And so...it is not really preference. (P12, female)

If they can come and we can explain, that would be very good. But most of the time, the residents' family cannot...come. And even (if) you talk over the phone...about all these small complex things... (when) their family (member) are not educated (in the medical field, and) you try to explain all these over the phone...it is very difficult. (D11, female)

\section{Subtheme: lack of coordination between health professionals in hospitals and nursing homes}

...if this patient is a complex patient that is seeing a lot of specialists in a hospital, I do not have that amount of information and really, I should not be the one to end up prescribing-deprescribing because I do not have enough information for the complex patient...usually I can just cancel (for medications prescribed by general practice)...whereas (on) the specialist side, I do not have enough information on my side and the family probably still prefer to listen to the specialist, which is rightfully so. (D11, female) This was an important point, as it signified that specialists have a major influence on GPs' autonomy and competence when considering stopping medications. Thus, GPs are more reluctant to change medications started by specialists.

Doctors also stated that deprescribing should begin at the hospital before discharge to the nursing homes. In particular, receptiveness by other doctors towards deprescribing, as well as receptiveness by other healthcare institutions following up with the patients (GPs and specialists) were deemed as important steps to improve deprescribing practice.

But when they are admitted, everything goes back to square one...because it is prescribed...the prescription actually arrives from the hospital before they are discharged. And once they are discharged, 
immediately there (should be) a suggestion to discontinue...or reduce. (D5, male)

\section{Subtheme: limited tools for deprescribing}

The most common deprescribing screening criteria known by doctors and pharmacists were the Screening Tool to Alert doctors to Right Treatment/Screening Tool of Older Person's Prescriptions (START/STOPP) criteria, ${ }^{19}$ as well as the Beers criteria, ${ }^{20}$ but most health professionals found them to be too stringent to be practical for the patients. They reported that they seldom referred to these tools but noted that these were useful guidelines.

A standard guideline that would help, because we have so many pharmacists with different ways of practicing and different habits. (P12, female)

Nurses claimed to follow doctors' and pharmacists' recommendations and rely on laboratory results rather than initiate deprescribing.

Usually, I am also reading the notes of the pharmacist or...if the doctors are doing laboratory tests... we are just waiting...for the next monthly (input) from the doctor... (and) for the next lab test. (N13, male)

\section{DISCUSSION}

There are a few enablers to deprescribing that were uncovered in this study. First, our findings suggest an improved deprescribing procedure and algorithm can facilitate deprescribing practice in nursing homes. Turner et al had similarly identified a need to standardise the process of deprescribing. ${ }^{12}$

Our study also highlighted that most participants, in particular nurses and pharmacists, agreed that multidisciplinary effort between doctors, pharmacists and nurses in the nursing homes is an important enabler in deprescribing. Unfortunately, unlike acute care hospitals, pharmacists and doctors are usually not around in the nursing homes most of the time, which may hinder communication. As such, this aspect can be one of the areas which can be improved, ${ }^{21}$ such as establishing a mechanism for face-to-face communications between doctors and pharmacists. In addition, our results also reflect that mentoring and case studies may also be helpful to increase the healthcare professional's confidence, especially among nurses, where knowledge and experience in deprescribing may be lacking.

Medication favoured for deprescribing by doctors and pharmacists are similar to findings from a Canadian Delphi consensus, where benzodiazepines, statins and proton pump inhibitors were identified, corresponding to mental health, cardiovascular and gastroenterological conditions. ${ }^{22}$ In addition, our study highlighted first-generation antihistamine as a prioritised class for deprescribing in our Asian setting. It was also commented in our study that a lot of patients are on good diet control in the nursing homes and their diabetes and hypertension may be well-controlled without the need for these medications.

The study also noted several challenges to deprescribing. First, we found psychotropic class of medication rarely gets reviewed by doctors as they are usually prescribed by the consultants. Studies have found that doctors expressed reluctance to interfere with medication prescribed by a colleague or medication specialist, possibly due to a lack of confidence in deprescribing skills and fear of litigation or conflict. ${ }^{623}$ Doctors in our study similarly expressed reluctance to deprescribe medication prescribed by consultants. One of the solutions could be to have a better communication channel between specialists, doctors and pharmacists with the institutions, consistent with a New Zealand GP study. ${ }^{10}$ With the recent launch of the nationwide Nursing Home IT Enablement Programme in Singapore that focused on incorporating patient management and electronic medical record from hospitals and polyclinics with nursing homes, this challenge may be reduced in future.

Second, doctors and pharmacists felt that under-reporting of adverse drug reactions might be common, given that many patients have communicative issues and taking the symptoms as part of the ageing process. Palagyi et al had similarly reported a lack of recognition in medication-related adverse drug reactions in both residents and their relatives, including the well-established increased risk of falls as well as impaired physical and cognitive function. ${ }^{6}$ However, in our study, nurses felt under-reporting is rare, given that they are by the side of the patients most of the time.

Third, the patient's preference seemed to take less precedence over patient's functional status (eg, ability to swallow) in deciding treatment selection. Other contributing factors include the inability to communicate and limited visitation by next-of-kins being contributing factors to making deprescribing preferences. Furthermore, pharmacists seldom have direct contact with patients and their treatment selections are determined primarily by nurses' feedback, as doctors are not always present. These may have deliberated deprescribing which would have otherwise taken place, as shared decision-making is lacking. However, it was also noted by others that shared decision-making may not be always possible in this setting. For example, Weir et al have identified that while some older adults preferred a proactive role in decision-making, others preferred to leave the decisions to their doctors. ${ }^{24}$

Lastly, our study found that most doctors and pharmacists were aware of START/STOPP criteria, ${ }^{19}$ as well as the Beers criteria, ${ }^{20}$ but most found these guidelines to be too stringent for deprescribing, making changes that are too impractical for an older patient. Our results supported the findings from a study by Ailabouni $e t a l$, which highlighted that lack of access to user-friendly evidence-based guidelines as a challenge to GPs in New Zealand, ${ }^{10}$ thus emphasising the need of a criteria-based guideline more 
suited for our region. Despite an abundance of tools to assist with deprescribing, ${ }^{25}$ there was no indication of the use of other deprescribing tools during the interviews, except the Beers and START/STOPP criteria as well as the local deprescribing guide developed for proton pump inhibitors. ${ }^{18}$ Limited awareness of deprescribing tools may be partly attributed to this lack of awareness on deprescribing, since this topic has not been taught in medical, pharmacy, or nursing undergraduate education. This calls for additional professional continuing education, as well as for the medical community to increase the awareness of deprescribing among its members.

In general, we witnessed a consistent belief in the health professionals interviewed that deprescribing might be a priority for their patients, in which deprescribing can reduce pill burden, adverse drug reactions, drug interactions, medication cost, medication errors and improve quality of life. Similar to a study on Dutch GPs which found the deprescribing of preventive medication difficult due to a lack of risk-benefits information, ${ }^{26}$ findings from this study showed that most physicians focus on the risk-benefit ratio when considering deprescribing. Our findings support the notion that prescribing based on younger adults' guidelines may not be practical given the limited risk-benefit ratio in older adults. ${ }^{27}$ Conversely, this might further add to their pill burden and cost, impacting on their quality of life.

To our best knowledge, this is one of the first known qualitative interview in Asia studying the perceptions of deprescribing among health professionals in Singapore's nursing homes. Our results add to existing findings to improve the uptake of deprescribing in residential care settings and may be applicable to other healthcare settings. Our results confirmed previous findings that the risk-benefit ratio is an important determinant in deprescribing. ${ }^{10}$ Our results similarly evidenced that first-generation antihistamine is perceived as an important target for deprescribing in our setting. ${ }^{11}$ Anticholinergic and sedative drug exposure have been associated with poorer physical and cognitive functions, ${ }^{28}$ and deprescribing of unnecessary first-generation antihistamine would potentially improve outcomes for this frail population. However, our study further found that we need a better process for deprescribing in nursing homes in Singapore. Despite the existence of established tools such as Beers ${ }^{20}$ and START/ STOPP criteria, ${ }^{19}$ our studies identified areas for improvement such as more suitable tools for our setting, mentoring and case discussions, as well as better collaboration and communication in the process of deprescribing. Better explicit deprescribing tools and algorithms that are developed or adapted for the Asian setting for deprescribing may help in greater practicability and comprehensiveness. We also identified that a lack of coordination between health professionals in hospitals and nursing homes could possibly hinder successful deprescribing in Singapore nursing homes. Future initiatives should look at increasing collaboration and communication between acute hospitals, nursing homes and specialist clinics in
Singapore. Future initiatives in Singapore can also look at educating health professionals in nursing homes on how to deprescribe and monitor in older adults.

There are several limitations to this study. Although we achieved saturation, there is a limited number of doctors and pharmacists available to participate in this study, as there is usually only one pharmacist and a handful of doctors covering each home, thus it may not be a true representative of all the healthcare workers working in the nursing homes. We acknowledged that most of the data could have been coded came from nurses. This may have an effect on displaying a balanced view of deprescribing from all included parties. We took this into consideration and reported any varied view from doctors, pharmacists and nurses separately in the subthemes. The fact that it was conducted face-to-face with the interviewer (who is a pharmacist) and being audio-recorded may give rise to biases in their answering of the questions. Although the deprescribing study had yet to commence, there is also a possibility that results of the study could be more biassed towards those who were already aware of the deprescribing study and thus had more motivation and interest in conducting deprescribing.

In conclusion, this study highlighted that deprescribing in the nursing homes is perceived by health professionals to be challenging and future research could assess how routine case studies, mentoring and better multidisciplinary communication could improve deprescribing knowledge and process in the nursing homes. Future studies should also explore patients' perspectives toward deprescribing in other parts of the world.

Acknowledgements The authors would like to thank Associate Professor lan Leong, Tan Tock Seng Hospital Singapore, for his kind assistance in devising the guiding questions for the interview. Study protocol 'Kua C, Yeo CYY, Char CWT, et al. Nursing home team-care deprescribing study: a stepped-wedge randomised controlled trial protocol. BMJ Open 2017;7:e015293. doi: 10.1136/ bmjopen-2016-015293'

Contributors C-HK contributed to the concept and design, data acquisition, analysis, interpretation for the work and writing drafts. SWHL and VM contributed to the design of the work (including analysis plan), interpretation of the data and revising the work critically for important intellectual content. All authors approved the version to be published.

Funding The authors have not declared a specific grant for this research from any funding agency in the public, commercial or not-for-profit sectors.

Competing interests None declared.

Patient consent for publication Not required.

Ethics approval Ethics approval was granted by Domain Specific Review Board of National Healthcare Group, Singapore (2016/00422) and Monash University Human Research Ethics Committee (2016-1430-7791).

Provenance and peer review Not commissioned; externally peer reviewed.

Data availability statement № data are available.

Open access This is an open access article distributed in accordance with the Creative Commons Attribution Non Commercial (CC BY-NC 4.0) license, which permits others to distribute, remix, adapt, build upon this work non-commercially, and license their derivative works on different terms, provided the original work is properly cited, appropriate credit is given, any changes made indicated, and the use is non-commercial. See: http://creativecommons.org/licenses/by-nc/4.0/.

ORCID iD

Shaun Wen Huey Lee http://orcid.org/0000-0001-7361-6576 


\section{REFERENCES}

1 Mitchell P, Koch T. An attempt to give nursing home residents a voice in the quality improvement process: the challenge of frailty. $J$ Clin Nurs 1997;6:453-61.

2 Ferreira AR, Martins S, Fernandes L. Comorbidity and polypharmacy in elderly living in nursing homes. European Psychiatry 2016;33:S471-2

3 Jokanovic N, Tan ECK, Dooley MJ, et al. Prevalence and factors associated with polypharmacy in long-term care facilities: a systematic review. J Am Med Dir Assoc 2015;16:535.e1-535.e12.

4 Lee SWH, Chong CS, Chong DWK. Identifying and addressing drugrelated problems in nursing homes: an unmet need in Malaysia? Int Clin Pract 2016;70:512.

5 Maher RL, Hanlon J, Hajjar ER. Clinical consequences of polypharmacy in elderly. Expert Opin Drug Saf 2014;13:57-65.

6 Palagyi A, Keay L, Harper J, et al. Barricades and brickwalls--a qualitative study exploring perceptions of medication use and deprescribing in long-term care. BMC Geriatr 2016;16:15.

7 Woodward MC. Deprescribing: achieving better health outcomes for older people through reducing medications. J Pharm Pract Res 2003;33:323-8

8 Lee SWH, Mak VSL. Changing demographics in Asia: a case for enhanced pharmacy services to be provided to nursing homes. $J$ Pharm Pract Res 2016;46:152-5.

9 Kua C-H, Mak VSL, Huey Lee SW. Health outcomes of deprescribing interventions among older residents in nursing homes: a systematic review and meta-analysis. J Am Med Dir Assoc 2019;20:362-72.

10 Ailabouni NJ, Nishtala PS, Mangin D, et al. Challenges and enablers of deprescribing: a general practitioner perspective. PLoS One 2016;11:e0151066

11 Kouladjian L, Gnjidic D, Reeve E, et al. Health care practitioners' perspectives on deprescribing anticholinergic and sedative medications in older adults. Ann Pharmacother 2016;50:625-36.

12 Turner JP, Edwards S, Stanners M, et al. What factors are important for deprescribing in Australian long-term care facilities? perspectives of residents and health professionals. BMJ Open 2016;6:e009781.

13 Bolmsjö BB, Palagyi A, Keay L, et al. Factors influencing deprescribing for residents in advanced care facilities: insights from general practitioners in Australia and Sweden. BMC Fam Pract 2016;17:152.

14 Anderson K, Stowasser D, Freeman C, et al. Prescriber barriers and enablers to minimising potentially inappropriate medications in adults: a systematic review and thematic synthesis. BMJ Open 2014;4:e006544.
15 Kua C-H, Yeo CYY, Char CWT, et al. Nursing home team-care deprescribing study: a stepped-wedge randomised controlled trial protocol. BMJ Open 2017;7:e015293.

16 Gale NK, Heath G, Cameron E, et al. Using the framework method for the analysis of qualitative data in multi-disciplinary health research. BMC Med Res Methodol 2013;13:117.

17 O'Brien BC, Harris IB, Beckman TJ, et al. Standards for reporting qualitative research: a synthesis of recommendations. Acad Med 2014;89:1245-51.

18 Pharmaceutical Society of Singapore. Pharmacy week 2016. Available: https://www.pss.org.sg/sites/default/files/PW/PW16/ deprescribing_slides_2016.pptx [Accessed 10 Feb 2019].

19 O'Mahony D, O'Sullivan D, Byrne S, et al. STOPP/START criteria for potentially inappropriate prescribing in older people: version 2. Age Ageing 2015;44:213-8.

20 Fick DM, Semla TP,By the American Geriatrics Society 2015 Beers Criteria Update Expert Panel . American geriatrics Society 2015 updated beers criteria for potentially inappropriate medication use in older adults. J Am Geriatr Soc 2015;63:2227-46.

21 Mak VSL, Lee SWH, March G. Pharmacists' roles in nursing homes in Malaysia. J Pharm Pract Res 2018;48:493-494.

22 Farrell B, Tsang C, Raman-Wilms L, et al. What are priorities for deprescribing for elderly patients? capturing the voice of practitioners: a modified Delphi process. PLoS One 2015;10:e0122246.

23 Pype P, Mertens F, Helewaut F, et al. Potentially inappropriate medication in primary care at the end of life: a mixed-method study. Acta Clin Belg 2018;73:213-9.

24 Weir K, Nickel B, Naganathan V, et al. Decision-Making preferences and deprescribing: perspectives of older adults and companions about their medicines. J Gerontol B Psychol Sci Soc Sci 2018;73:e98-107.

25 Motter FR, Fritzen JS, Hilmer SN, et al. Potentially inappropriate medication in the elderly: a systematic review of validated explicit criteria. Eur J Clin Pharmacol 2018;74:679-700.

26 Schuling J, Gebben H, Veehof LJG, et al. Deprescribing medication in very elderly patients with multimorbidity: the view of Dutch GPs. A qualitative study. BMC Fam Pract 2012;13:56.

27 Naganathan V. Cardiovascular drugs in older people. Aust Prescr 2013;36:190-4.

28 Hilmer SN, Mager DE, Simonsick EM, et al. A drug burden index to define the functional burden of medications in older people. Arch Intern Med 2007;167:781-7. 